\title{
A DEPENDENCE BETWEEN OPTICAL AND RADIO LUMINOSITIES OF QUASARS AFTER ALL?
}

\author{
Jerzy Machalsixi \\ Astronomical Observatory, Jagellonian University \\ ul. Orla 171, \\ PL-30244 Cracow, \\ Poland.
}

It is still uncertain whether optical anc radio emission mechanisms in AGNs are coupled or not, and how? New observational data give more light on this subject. The data used in this analysis are shown in Table I:

\begin{tabular}{lrllll}
\hline Sample & ir & $\begin{array}{l}\text { Siry area } \\
(\mathrm{sr})\end{array}$ & $\begin{array}{c}\text { S } \\
(\mathrm{Jy})\end{array}$ & $\begin{array}{l}\text { B-(J-) } \\
\text { photometry }\end{array}$ & $\begin{array}{l}\text { Red- } \\
\text { shift }\end{array}$ \\
\hline BDFL & 68 & 4.30 & $>2.0$ & $41(60 \%)$ & $68(100 \%)$ \\
GB/GB2 & 83 & $0.44 / 0.091 *$ & $0.25 \div 2.0$ & $16(19 \%)$ (UBVRI) & $29(35 \%)$ \\
LBDS & 25 & 0.0055 & 20.001 & $25(100 \%)$ (UJFN) & $5(25 \%)$ \\
VLA & 5 & 0.0012 & 20.005 & - & - \\
\hline
\end{tabular}

* $0.44 \mathrm{sr}$ for $\mathrm{S} \geq 0.55 \mathrm{Jy} ; 0.091 \mathrm{sr}$ for $\mathrm{S}<0.55 \mathrm{Jy}$

Apparent blue ( $B$ or $J$ ) magnitude of these objects plotted against their $1.4 \mathrm{GHz}$ flux densities (Machalski and Condon $1985)$ suggested a positive, though weak, $\operatorname{logF}(2500 \AA)-\log P$ (1400) correlation. Since all samples but one were uncomplete in redshifts, the mean redshifts are estimated for them with two independent statistical approaches employing:

1. the $\Psi(R)$ function of Fanti and Perola (1977) (assumed to be known for logR 3.5, and the optical-luminosity $\{\Phi(F)\}$ and evolution $\{\mathrm{E}(\mathrm{z})\}$ functions of Bahcall and Turner (1977); 2. numerical experiments to generate a probable redshift for each QSO candidate, concordant with the observed Hubble diagram for QSOs.

For each QSO sample complete over A(sr), the number of expected optical quasars (if their $\Phi(F)$ anc $E(z)$ functions are known) was compared with the observed number of objects having a specified radio/optical luminosity ratio $R$. A redshift corresponding to a median distance (redshift) within which a half of the observed objects $\mathbb{N}(<\mathrm{m}, \mathrm{r})$ should lay is the resultant median redshift. This is shown in Table II 
along with corresponding optical and radio luminosities for different samples considered.

\begin{tabular}{lllll}
\hline Sample & \multicolumn{1}{c}{$\begin{array}{c}\mathrm{logF}(2500) \\
(\text { mediar })\end{array}$} & $\begin{array}{c}\text { logP }(1400) \\
(\mathrm{Hz})\end{array}$ & $\alpha_{\text {ro }}$ \\
\hline BDFL & $0.86 \pm 0.07 *$ & $23.24 \pm 0.07 *$ & $27.44 \pm 0.06 *$ & 0.71 \\
GB $(0.55)$ & $1.18 \pm 0.12$ & $23.14 \pm 0.14$ & $26.99 \pm 0.11$ & 0.65 \\
GB $(0.25)$ & $1.22 \pm 0.14$ & $23.06 \pm 0.16$ & $26.79 \pm 0.13$ & 0.63 \\
LBDS & $1.48 \pm 0.30 *$ & $22.91 \pm 0.30 *$ & $25.85 \pm 0.31 *$ & 0.50 \\
\hline
\end{tabular}

* observed values (in. LBDS sample for five objects only)

The above trend was confirmed with the numerical experiments. An example is shown in Fig. 1 (left):
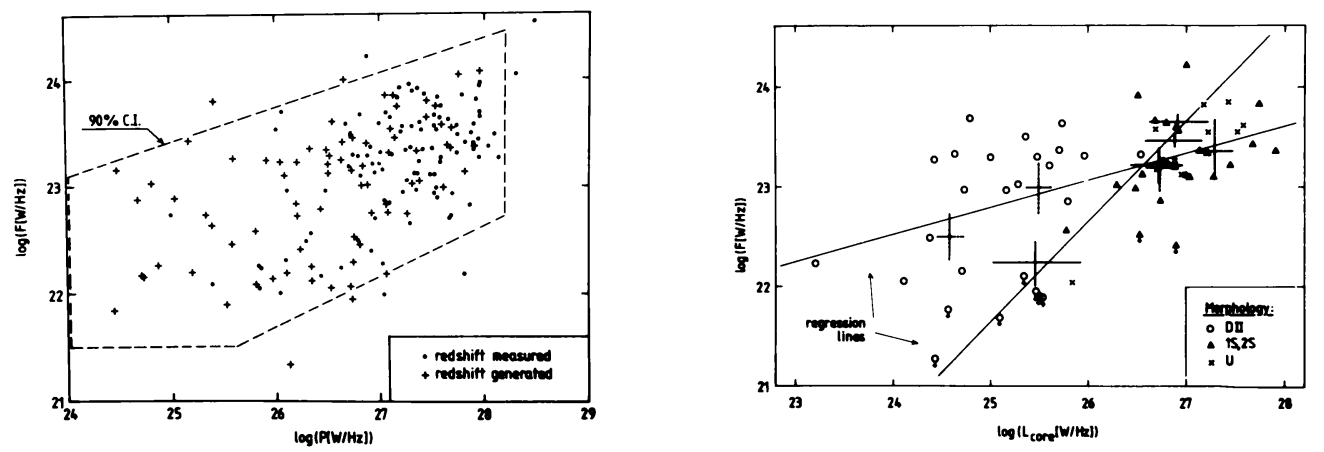

$90 \%$ confidence intervals (C.I.) for the mean logF in cifferent logP bins bound an area marked in Fig. 1. A logF-logP dependence seen in Table I and Fig. 1 was verified using the Spearman partial rank correlation test to examine an influence of the logF-z and logP-z correlations, arisec mainly from selection effects. The test showed that the correlation coefficient between $\log F$ and logP remains about 0.5 . This result is significant at the 50 level. A similar correlation was found between nuclear luminosities in the BDFL and GB/GB2 samples (shown in Fig. 2; right). Since the apparent logF-logP correlation is mainly caused by faint LBDS+VLA objects, it suggests that most of these less luminous quasars can be rather compact. If there was true, less luminous radio-selected QSOs would have smaller intrinsic size than do bright ciuasars. It is possible that a negative correlation between radio luminosity and size (cf. Macklin 1982; and references therein) concerns only very luninous quasars.

REFERENCES

Bahcall, J.N., and Turner, R.L. (1977). IAU Symp. No. 74, p. 295 Fanti, R., and Perola, G.C. $(1977)$. IAU Symp. No. 74, p. 171 Machalski, J., and Condon, J.J.(1985).A.J. (submitted) Macklin, J.T. (1982). M.I.R.A.S. 199, $\frac{11119}{119}$ 